\title{
Transient analysis of thermal and moisture transfer in building materials
}

\author{
Haibo Wang ${ }^{1}$, Xingguo Guo ${ }^{2}$ \\ ${ }^{1}$ School of Architecture and Traffic Engineering, Ningbo University of Technology, Ningbo \\ 315211, China \\ ${ }^{2}$ School of Civil Engineering and Architecture, Nanchang University, Nanchang 330031, China
}

Email:467062043@qq.com

\begin{abstract}
This paper aims to identify the transient temperature and humidity distribution during the coupled heat and moisture transfer in porous building materials. To this end, the Laplace transform and finite-difference method were combined into a hybrid numerical method, after the preliminary discussion on the coupled heat and moisture transfer in building materials. Then, the governing equations of heat and moisture transfer in porous materials were solved by the hybrid numerical method. Under the boundary conditions, the general solution of the governing equation was obtained considering the coupling of temperature and humidity on the inner and outer surfaces of building materials. The inversion of the real domain was accomplished by matrix operation and Fourier sequence. The research provides practical methods and empirical data for further research and engineering practice, especially for buildings in humid regions.
\end{abstract}

Keywords: Hybrid Numerical Method, Coupled Heat and Moisture Transfer, Transient Analysis.

\section{INTRODUCTION}

Most building materials are water-bearing porous materials, such as concrete, wood, and so on. The moisture transfer in these materials poses a great impact on indoor humidity, airconditioning load, and the cooling load [1, 2]. In most building materials, there is a coupling effect between temperature and humidity, especially when the ratio of thermal conductivity to water diffusivity is close to the unit value. Despite the intensive research [3-8] on the interaction between temperature and humidity, most scholars have only discussed the ideal case under boundary conditions. Moreover, the existing research is concentrated on the coupling effect in the material, failing to tackle the energy and mass balance on some boundaries [9].

In recent years, great progress has been made in the solution of heat and mass transfer equations. For instance, Mikhailov and Ozisik [10] derived the analytical solution of linear problem based on the traditional integral transformation method. Nevertheless, some complex eigenvalues were discovered and found to influence the distribution of temperature and humidity [11]. Chang et al. [14] employed decoupling technique to couple the governing equations, but did not realize the simultaneous positioning of the coupled governing equations and boundary conditions. Cheroto et al. [7] proposed an improved lumped system analysis method to produce approximate solutions. This approach avoids complex empirical formulas at the cost of accuracy. Taking physical models as semi-infinite media, Fudym [12] put forward a 1D linear equation for unsteady coupled heat and moisture transfer, and give the solution to the equation.

In actual practice, some scholars have made assumptions to obtain the analytical solutions of the equations, such as regarding the total pressure as a constant and viewing the physical parameters of the material as fixed. Taking the partial pressure and temperature of water vapour as driving potential, Chen Youming and Chen Zaikang established the equations of simultaneous heat and moisture transfer for porous building materials in the isothermal moisture absorption and desorption range, and discussed the conditions for linearization of the equations. Guo Xingguo set up a new model of coupled heat and moisture transfer in a multilayer wall, taking the humidity and temperature of the air in the wall as the driving force, and discretized the governing equations by the finite-difference method. In his model, the moisture content of the air is considered as a function of the moisture content and temperature of the materials.

In light of the above, this paper introduces a hybrid numerical method to discuss the transient temperature and humidity distribution during the coupled heat and moisture transfer in building materials. First, the general solution of the governing equations was obtained in the transform domain by Laplace transform and the finite-difference method. Then, the real domain was inversed by matrix operation and Fourier series. 


\section{DERIVATION OF GOVERNING EQUATIONS}

Under temperature gradient, humidity gradient, capillary force, pressure and gravity, heat and moisture transfer may occur in porous building materials at the coupling of the internal temperature field and humidity field. The transfer process involves the heat conduction of fluid, the naturalconvection heat transfer of fluid, the latent heat transfer in the phase change of gas and liquid, as well as the convective mass transfer caused by molecular diffusion and macroscopic motion of fluid between solid particles and pores.

Before exploring the heat and moisture transfer in waterbearing porous materials, it is necessary to describe the process with heat and mass transfer equations. In general, the fluid movement must obey the law of conservation of mass and the law of conservation of energy. Therefore, the basic equations should contain the mass conservation equation and the energy conservation equation.

The conservation equations depict the energy and mass changes. The energy or mass flowing in or out through the medium surface per unit of time equals the enthalpy or humidity variation of the medium. Below is the energy conservation equation:

$\frac{\partial H}{\partial \mathrm{t}}=-\nabla q+S_{h}$

where $\mathrm{H}$ is the total enthalpy $\left(\mathrm{J} / \mathrm{m}^{3}\right)$; qis the heat flux density $\left(\mathrm{w} / \mathrm{m}^{2}\right) ; \mathrm{S}_{\mathrm{h}}$ is the source or sink of heat $\left(\mathrm{w} / \mathrm{m}^{3}\right)$.

The total enthalpy equals the sum of the enthalpy of dry material and that of moisture inside the material.

$$
H=H_{\mathrm{s}}+H_{\mathrm{w}}
$$

where $\mathrm{H}_{\mathrm{s}}$ is the enthalpy of dry material $\left(\mathrm{J} / \mathrm{m}^{3}\right) ; \mathrm{H}_{\mathrm{w}}$ is the enthalpy of moisture inside the material $\left(\mathrm{J} / \mathrm{m}^{3}\right)$.

The heat flux density is proportional to the thermal conductivity and the temperature gradient of the water-bearing material.

$$
q=-k \nabla T
$$

where $\mathrm{k}$ is the thermal conductivity of water-bearing material $(\mathrm{w} /(\mathrm{m} \cdot \mathrm{k}))$; $\mathrm{T}$ is the absolute temperature $(\mathrm{K})$.

The heat generated from phase change is often considered as a source term in the energy conservation equation. Meanwhile, the heat of absorption or desorption of the material is also considered as a source term. Therefore, the source term of the energy conservation equation is obtained as follows.

$$
S_{h}=-\left(\sigma h_{l v}+\gamma\right) \frac{\partial w}{\partial t}
$$

where $S_{h}$ is the heat source or sink resulted from condensation or evaporation $\left(\mathrm{J} /\left(\mathrm{m}^{3} \cdot \mathrm{s}\right)\right)$; $\mathrm{h}_{\mathrm{lv}}$ is the latent heat of phase change $(\mathrm{J} / \mathrm{kg}) ; \sigma$ is the phase change factor; $\gamma$ is the heat of absorption or desorption $(\mathrm{J} / \mathrm{kg}) ; \mathrm{w}$ is the water content $\left(\mathrm{kg} / \mathrm{m}^{3}\right)$.

Similar to the energy conservation equation, the mass conservation equation of moisture is expressed as: $\frac{\partial w}{\partial t}=-\nabla\left(g_{w}+g_{v}\right)+s_{w}$

where $\mathrm{g}_{\mathrm{w}}$ is the transmission flux density of liquid $\left(\mathrm{kg} /\left(\mathrm{m}^{2} \cdot \mathrm{s}\right)\right)$; $\mathrm{g}_{\mathrm{v}}$ is the transmission flux density of gas $\left(\mathrm{kg} /\left(\mathrm{m}^{2} \cdot \mathrm{s}\right)\right)$; $\mathrm{S}_{\mathrm{w}}$ is the source or sink of moisture $\left(\mathrm{kg} /\left(\mathrm{m}^{2} \cdot \mathrm{s}\right)\right)$.

The transmission flux density of liquid depends on the relative humidity gradient.

$$
g_{w}=-D_{\phi} \nabla \phi
$$

where $D_{\varphi}$ is the conductivity coefficient of liquid $(\mathrm{kg} /(\mathrm{m} \cdot \mathrm{s}))$; $\varphi$ is the relative humidity.

Then, the diffusion flux density of water vapour is:

$$
g_{v}=-\delta_{p} \nabla p
$$

where $\delta_{\mathrm{p}}$ is the water vapour permeability in building material $(\mathrm{kg} /(\mathrm{m} \cdot \mathrm{s} \cdot \mathrm{Pa})) ; \mathrm{p}$ is the partial pressure of vapour $(\mathrm{Pa})$. The water vapour permeability in building material equals the ratio of the water vapour permeability in the stagnant air to the diffusion resistance factor of water vapour.

The source term of moisture in building material is so few as to negligible here. The sink term of moisture depicts the solidification of concrete and mortar. It is also ignored in this paper because solidification, as a chemical process, falls out of our research scope.

Obviously, the energy conservation equation is closely coupled with the mass conservation equation. The moisture content in the material affects the thermal conductivity and the source term. In return, the temperature also influences the moisture transfer process. In summary, the equations for coupled heat and moisture transfer are as follows:

$$
\begin{aligned}
& \frac{d H}{d t}=\nabla \bullet(\lambda \nabla T)+\left(\zeta h_{l v}+\gamma\right) \frac{\partial w}{\partial t} \\
& \frac{\partial w}{\partial t}=\nabla \bullet\left[D_{\phi} \nabla \phi+\delta_{p} \nabla\left(\phi p_{s a t}\right)\right]
\end{aligned}
$$

where $\mathrm{p}_{\text {sat }}$ is the saturation pressure of water vapour $(\mathrm{Pa})$.

$$
\begin{aligned}
& \frac{\partial w}{\partial t}=\frac{\partial w}{\partial \phi} \frac{\partial \phi}{\partial t}=\rho \frac{\partial w}{\partial \phi} \frac{\partial v}{\rho v_{t} \partial t} \\
& C_{m}=\frac{1}{\rho v_{t}} \frac{\partial w}{\partial \phi}
\end{aligned}
$$

where $\mathrm{C}_{\mathrm{m}}$ is the specific humidity; $\frac{d_{w}}{d_{\varphi}}$ is the slope of the isothermal adsorption line.

$\frac{\partial w}{\partial t}=\rho C_{m} \frac{\partial v}{\partial t}$

Then, formula (8) can be converted as:

$\rho C_{p} \frac{d T}{d t}=\nabla \bullet(\lambda \nabla T)+\rho C_{m}\left(\zeta h_{l v}+\gamma\right) \frac{\partial v}{\partial t}$ 
Whereas the saturation pressure is a single-valued function of temperature, the following formula can be derived:

$$
\begin{aligned}
& \frac{\partial w}{\partial t}=\nabla \bullet\left[\lambda \nabla_{v}+v \nabla T\right] \\
& \frac{\partial w}{\partial t}=\rho C_{m} \frac{\partial v}{\partial t}=\nabla \bullet(\lambda \nabla v)+\nabla \bullet(v \nabla T)
\end{aligned}
$$

where $\lambda$ is the moisture diffusion coefficient $\left(\mathrm{m}^{2} / \mathrm{s}\right) ; \mathrm{v}$ is the diffusion coefficient induced by temperature gradient $\left(\mathrm{kg} /\left(\mathrm{m} \cdot \mathrm{s} \cdot{ }^{\circ} \mathrm{C}\right)\right)$.

The temperature diffusivity and the moisture diffusion coefficient are closely intertwined and difficult to differentiate through experiment. Therefore, the coefficient of temperature gradient was introduced to assist with the differentiation. This coefficient is expressed as:

$$
\zeta=\frac{v}{\lambda}
$$

\section{MATHEMATICAL MODEL}

\subsection{Governing equations}

Typical heat and mass transfer problems can be described by the Luikov equation [13]. In a recent study, 1D governing equations are established, considering the temperature and humidity in the wall and the effect of heat absorption and desorption. Here, the original equations are improved by changing the driving potential from temperature and moisture content to temperature and water vapour content. In this way, the author resolved the discontinuity of moisture content in porous media. In the meantime, the heat adsorption or desorption of porous media and the latent heat (evaporation or condensation) of water were taken as the heat source or heat sink, and added to the equations of coupled heat and moisture transfer. The phase change of water leads to the coupling between mass transfer and heat transfer.

For simplicity, the following assumption were put forward: (1) The solid, liquid and gas are continuous media, and in local thermodynamic equilibrium; (2) The building material is isotropic and the values of its physical parameters are constant; (3) the pressure of moist air in the material is constant, and the water vapour and air are ideal gases; (4) the coupled heat and moisture transfer process is simplified as a 1D transfer process along the thickness direction of the wall, without considering the adsorption hysteresis; (5) the effect of material usage history on heat and moisture transfer is negligible, and the effect of temperature on the moisture of the material is also negligible; (6) the moisture in the material exists only in the forms of vapour and liquid. 15]:

The coupled governing equations can be expressed as [14-

$$
\begin{aligned}
& \rho C_{p} \frac{\partial T}{\partial t}=k \frac{\partial^{2} T}{\partial x^{2}}+\rho C_{m}\left(\varepsilon h_{l v}+\gamma\right) \frac{\partial m}{\partial t} \\
& \rho C_{m} \frac{\partial m}{\partial t}=D_{m} \frac{\partial^{2} m}{\partial x^{2}}+D_{m} \delta \frac{\partial^{2} T}{\partial x^{2}}
\end{aligned}
$$

where $\mathrm{T}$ is the temperature; $\mathrm{m}$ is the moisture potential; $\mathrm{k}$ is the thermal conductivity coefficient; $\mathrm{D}_{\mathrm{m}}$ is the moisture diffusivity coefficient; $\mathrm{C}_{\mathrm{p}}$ is the heat capacity of the material; $\mathrm{C}_{\mathrm{m}}$ is the moisture content of the material; $\rho$ is the material density; $h_{l v}$ is the latent heat of material; $\gamma$ is the heat of absorption or desorption; $\delta$ is the thermal gradient coefficient; $\varepsilon$ is the ratio of the vapour diffusion coefficient to the total moisture diffusivity.

All the material properties mentioned above are effective properties. The moisture potential depends on the moisture content. The dependence can be expressed as: $\mathrm{C}=\mathrm{C}_{\mathrm{m}} \mathrm{m}$

The governing formulas (17) and (18) contain both general diffusion equations and some source or sink terms. The governing formula (17) expresses the thermal energy balance in the wall. The last term in this formula represents the heat source or heat sink for the fluid-vapour phase change and heat absorption or desorption. Similarly, the governing formula (18) expresses the water balance in the wall. The last term in this formula depicts the wet source or wet sink resulted from the temperature gradient.

Divide formula (17) by $\rho C_{p}$ and substitute the quotient to formula (18), and we have:

$L \frac{\partial^{2} T}{\partial x^{2}}=\frac{\partial T}{\partial t}-v \frac{\partial m}{\partial t}$

$D \frac{\partial^{2} m}{\partial x^{2}}=\frac{\partial m}{\partial t}-\lambda \frac{\partial T}{\partial t}$

where $L=\frac{k}{\rho C_{p}} ; D=\frac{k D_{m}}{\rho C_{m}\left[k+D_{m} \delta\left(\varepsilon h_{l v}+\gamma\right)\right]}$

$v=\frac{C_{m}\left(\varepsilon h_{l v}+\gamma\right)}{C_{p}} ; \lambda=\frac{C_{p} D_{m} \delta}{C_{m}\left[k+D_{m} \delta\left(\varepsilon h_{l v}+\gamma\right)\right]}$

$\mathrm{v}$ and $\lambda$ are the equivalent thermal conductivity coefficient and the equivalent water diffusivity, respectively. The coefficients are usually positive. The moisture in formula (19) is the heat source of the temperature distribution is the heat source of temperature distribution if the moisture absorption rate is positive $\left(\frac{\partial_{m}}{\partial_{t}}>0\right)$, and the heat sink of temperature distribution if the moisture rate is negative $\left(\frac{\partial_{m}}{\partial_{t}}<0\right)$. Similarly, the temperature can also serve as a wet source or a wet sink depending on the positivity or negativity of the temperature rate.

\subsection{Boundary conditions}

The diffusion of mass and the transfer of heat are mutually affected during the coupled heat and mass transfer process in porous media. In other words, the latent heat of water joins the energy conservation, and the mass diffusion induced by temperature and humidity gradient also affects the mass conservation. For the classical problem of coupled heat and moisture transfer in porous media, the boundary conditions are as follows:

$$
\begin{aligned}
& k \frac{\partial T\left(x_{1}, t\right)}{\partial x}=h_{c 1}\left[T\left(x_{1}, t\right)-T_{\infty 1}\right]+ \\
& (1-\varepsilon) h_{l v} h_{m 1}\left[m\left(x_{1}, t\right)-m_{\infty 1}\right]
\end{aligned}
$$


$-k \frac{\partial T\left(x_{2}, t\right)}{\partial x}=h_{c 2}\left[T\left(x_{2}, t\right)-T_{\infty 2}\right]+$

$(1-\varepsilon) h_{l v} h_{m 2}\left[m\left(x_{2}, t\right)-m_{\infty 2}\right]$

$D_{m} \frac{\partial m\left(x_{1}, t\right)}{\partial x}+D_{m} \delta \frac{\partial T\left(x_{1}, t\right)}{\partial x}=h_{m 1}\left[m\left(x_{1}, t\right)-m_{\infty 1}\right]$

$-D_{m} \frac{\partial m\left(x_{2}, t\right)}{\partial x}-D_{m} \delta \frac{\partial T\left(x_{2}, t\right)}{\partial x}=h_{m 2}\left[m\left(x_{2}, t\right)-m_{\infty 2}\right]$

where $h_{c}$ is the convective heat transfer coefficient $\left(\mathrm{w} /\left(\mathrm{m}^{2} \cdot \mathrm{k}\right)\right)$; $\mathrm{h}_{\mathrm{m}}$ is the convective moisture transfer coefficient $(\mathrm{m} / \mathrm{s})$.

Formulas (21) and (22) represent the heat conservation at the boundary. The two items on the right side of the formulas respectively stand for convection heat transfer and the latent heat of phase change. Formulas (23) and (24) represent the moisture convervation at the boundary. The two items on the left side of the formulas are respectively moisture flux under temperature gradient and the moisture gradient; the right side of the formulas represents the amount of vapour flowing out or into the surface under convective mass transfer. The initial temperature and the amount of water vapour in the porous media are defined as follows:

$T(x, 0)=T_{0}$

$m(x, 0)=m_{0}$

\section{SOLVING METHOD}

The coupled heat and moisture transfer in porous media is commonly solved by analytical method, the numerical method, and the simulation method. The analytic method includes direct integration, variable separation, image mapping, conformal mapping, superposition, Duhamel integration, small perturbation, Laplace transform and approximate integration. Despite strict inference and clear expression, the analytic method is limited to simple situations like linear homogeneous problems and simple nonlinear nonhomogenous ones.

The numerical method is the most popular way to handle the coupled heat and moisture transfer in porous media. The typical examples are finite-difference method, finite-element method, transfer function method, control volume method, boundary element method and effective thermal conductivity method. Compared with the analytic method, the numerical method does well in complex nonlinear and non-homogenous problems, and achieves desirable solutions as long as the discretization analysis is appropriate. In view of this, a mixed numerical method was created to solve our problem.

Through Laplace transform, the formulas (19), (20), (21), (22), (23) and (24) are converted into the following expressions:

$$
\begin{aligned}
& L \frac{d^{2} \bar{T}}{d x^{2}}=S \bar{T}-T_{0}-v S \bar{m}+v m_{0} \\
& D \frac{d^{2} \bar{m}}{d x^{2}}=S \bar{m}-m_{0}-\lambda S \bar{T}+\lambda T_{0} \\
& k \frac{d \bar{T}\left(x_{1}, S\right)}{d x}=h_{c 1}\left[\bar{T}\left(x_{1}, S\right)-\frac{T_{\infty 1}}{S}\right]+ \\
& (1-\varepsilon) h_{l v} h_{m 1}\left[\bar{m}\left(x_{1}, S\right)-\frac{m_{\infty 1}}{S}\right] \\
& -k \frac{d \bar{T}\left(x_{2}, S\right)}{d x}=h_{c 2}\left[\bar{T}\left(x_{2}, S\right)-\frac{T_{\infty 2}}{S}\right]+ \\
& (1-\varepsilon) h_{l v} h_{m 2}\left[\bar{m}\left(x_{2}, S\right)-\frac{\left.m_{\infty 2}\right]}{S}\right] \\
& D_{m} \frac{d \bar{m}\left(x_{1}, S\right)}{d x}+D_{m} \delta \frac{d \bar{T}\left(x_{1}, S\right)}{d x}= \\
& h_{m 1}\left[\bar{m}\left(x_{1}, S\right)-\frac{m_{\infty 1}}{S}\right] \\
& D_{m 2}\left[\frac{\left.d \bar{m}\left(x_{2}, S\right)-\frac{m_{\infty 2}}{S}\right]}{d \bar{m}\left(x_{2}, S\right)}-D{ }_{m} \delta \frac{d \bar{T}\left(x_{2}, S\right)}{d x}=\right. \\
& D_{m}
\end{aligned}
$$

where $\bar{T}(S)$ and $\bar{m}(S)$ are the Laplace transform of $\mathrm{T}(\mathrm{t})$ and $\mathrm{m}(\mathrm{t})$, respectively; $\mathrm{S}$ is the Laplace transform parameter. The following discretized equations can be obtained by finding the central finite difference of formulas (27) and (28):

$L \frac{\bar{T}_{j+1}-2 \bar{T}_{j}+\bar{T}_{j-1}}{\Delta x^{2}}-S \bar{T}_{j}+$

$v S \bar{m}_{j}=v m_{0}-T_{0}$

$D \frac{\bar{m}_{j+1}-2 \bar{m}_{j}+\bar{m}_{j-1}}{\Delta x^{2}}-S \bar{m}_{j}+$

$\lambda S \bar{T}_{j}=-m_{0}+\lambda T_{0}$

where $\Delta \mathrm{x}=\mathrm{l} /(\mathrm{N}-1) ; 1$ is the wall thickness; $\mathrm{N}$ is the total number of nodes.

Then, substitute the boundary conditions in formulas (29) (32) into formulas (33) and (34), and express the resulting formulas in matrix form:

$$
\begin{aligned}
& \{[A]-S[I]\}\left\{\bar{T}_{j}\right\}+\{[B]+v S[I]\}\left\{\bar{m}_{j}\right\}=\{C\} \\
& \{[F]+\lambda S[I]\}\left\{\bar{T}_{j}\right\}+\{[G]-S[I]\}\left\{\bar{m}_{j}\right\}=\{H\}
\end{aligned}
$$

where $[\mathrm{I}]$ is a unit matrix. 
$[A]=\left[\begin{array}{cccccc}-\frac{2 L}{\Delta x^{2}}-\frac{2 L h_{c 1}}{k \Delta x} & \frac{2 L}{\Delta x^{2}} & 0 & \cdots & 0 & 0 \\ \frac{L}{\Delta x^{2}} & -\frac{2 L}{\Delta x^{2} S} & \frac{L}{\Delta x^{2}} & \cdots & 0 & 0 \\ \vdots & \vdots & \vdots & \vdots & \vdots & \vdots \\ 0 & \cdots & \frac{L}{\Delta x^{2}} & -\frac{2 L}{\Delta x^{2}} & \frac{L}{\Delta x^{2}} \\ 0 & \cdots & 0 & \frac{2 L}{\Delta x^{2}} & -\frac{2 L}{\Delta x^{2}}-\frac{2 L h_{c 2}}{k \Delta x}\end{array}\right]$

$[G]=\left[\begin{array}{cccccc}-\frac{2 D}{\Delta x^{2}}+\frac{2 D \delta(1-\varepsilon) h_{l v} h_{m 1}}{k \Delta x}-\frac{2 h_{m 1} D}{\Delta x D_{m}} & \frac{2 D}{\Delta x^{2}} & 0 & \cdots & & 0 \\ \frac{D}{\Delta x^{2}} & -\frac{2 D}{\Delta x^{2}} & \frac{D}{\Delta x^{2}} & \cdots & & 0 \\ \vdots & \vdots & \vdots & \vdots & \vdots & \vdots \\ 0 & 0 & \cdots & \cdots & -\frac{2 D}{\Delta x^{2}} & \frac{D}{\Delta x^{2}} \\ 0 & 0 & \cdots & & \frac{2 D}{\Delta x^{2}} & -\frac{2 D}{\Delta x^{2}}+\frac{2 D \delta(1-\varepsilon) h_{l v} h_{m 2}}{k \Delta x}-\frac{2 h_{m 2} D}{\Delta x D_{m}}\end{array}\right]$

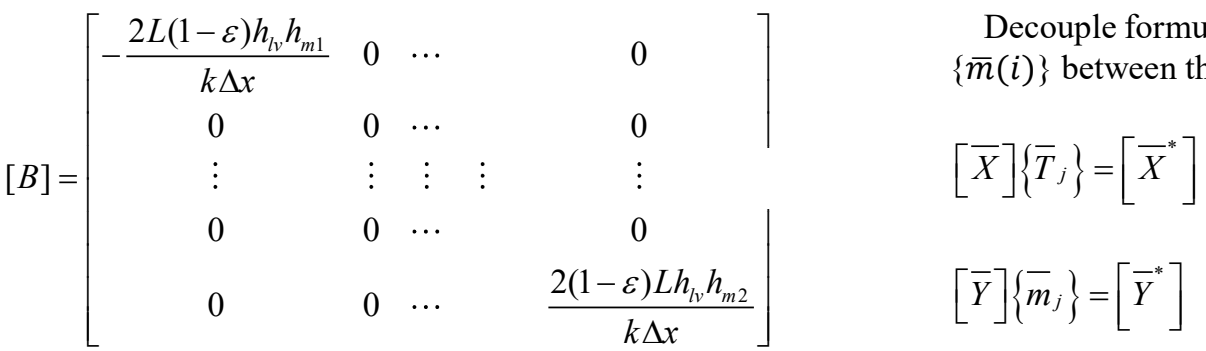

$[C]=\left[\begin{array}{c}v m_{0}-T_{0}-\frac{2 L h_{c 1} T_{\infty 1}}{k \Delta x S}-\frac{2 L(1-\varepsilon) h_{l v} h_{m 1} m_{\infty 1}}{k \Delta x S} \\ v m_{0}-T_{0} \\ \vdots \\ v m_{0}-T_{0} \\ v m_{0}-T_{0}-\frac{2 L h_{c 2} T_{\infty 2}}{k \Delta x S}-\frac{2 L(1-\varepsilon) h_{l v} h_{m 2} m_{\infty 2}}{k \Delta x S}\end{array}\right]$

where:

$[\bar{X}]=\{[F]+\lambda S[I]\}-\{[G]-S[I]\}\{[B]+v S[I]\}^{-1}\{[A]-S[I]\}$

$\left[\bar{X}^{*}\right]=\{[H]\}-\{[G]-S[I]\}\{[B]+v S[I]\}^{-1}\{[C]\}$

$[\bar{Y}]=\{[G]-S[I]\}-\{[F]+\lambda S[I]\}\{[A]-S[I]\}^{-1}\{[B]+v S[I]\}$

$\left[\bar{Y}^{*}\right]=\{[H]\}-\{[F]+\lambda S[I]\}\{[A]-S[I]\}^{-1}\{[C]\}$

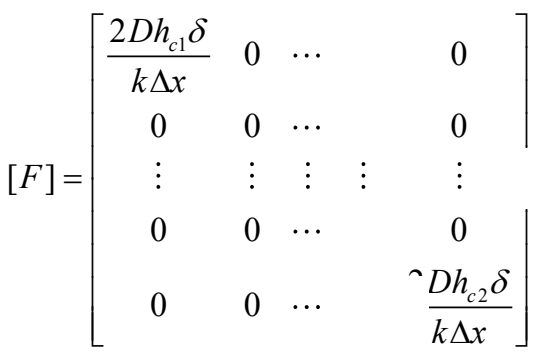

The Laplace inverse transformation of complex formulas (37) and (38) can be accomplished by residue theorem or Fourier series.

\section{CONCLUSION}

In this paper, the Laplace transform and finite-difference method are combined to find the transient temperature and humidity distribution of building materials. The hybrid numerical method combines the advantages of both components, and achieves high accuracy than each of them. The research provides practical methods and empirical data for further research and engineering practice, especially for buildings in humid regions. 


\section{REFERENCES}

[1] Wang Z.J. (2005). Discussion on thermal sensation and thermal comfort, Building Energy \& Environment, Vol. 24, No. 2, pp. 93-94.

[2] Liu X.Y., Zhen C.Y., Huang C.F. (2009), Analysis effect factor of thermal conductivity for porous materials, Low Temperature Architecture Technology, No. 9, pp. 121.

[3] Wu H.W., Wang H.H., Zhuang Y.Y. (2009). The application prospect of hygroscopic materials for construction of energy-saving, Refrigeration \& Air Condition, Vol. 23, No. 6, pp. 102-106. DOI: 10.3969/j.issn.1671-6612.2009.06.027

[4] Kong F.H., Zheng M.Y., Han Z.W., Yang T. (2008). Effect of heat and moisture transfer of new built building envelop on building energy consumption, Heating Ventilating \& Air Conditioning, Vol. 38, No. 7, pp. 6-7. DOI: 10.3969/j.issn.1002-8501.2008.07.002

[5] Martínez-Flores E.E. (2001). Construction and Building materials, Construction \& Building Materials, Vol. 15, No. 8, pp. 465-471. DOI: 10.1016/S0950$\underline{0618(01) 00021-6}$

[6] Guo X.G., Chen Y.M. (2008). Analysis of the hydrothermal performance of multilayer wall exposed to hot and humid climate, Journal of Hunan University (Natural Sciences), Vol. 35, No. 8, pp. 1-2.

[7] Liu W. (2008). Experimental study on the masonry brick of fly ash slag, Science and Technology of West China, Vol. 2b, pp. 20-21. DOI: 10.3969/j.issn.1671$\underline{6396.2005 .04 .014}$
[8] Chen Z.Y. (2010). Application and research review of humidity-controlling materials for building energy conservation, New Chemical Materials, Vol. 38, No. 7, pp. 20-22. DOI: 10.3969/j.issn.1006-3536.2010.07.007

[9] Lin Y.C. (2008). External wall insulation technology and energy-saving materials, Fujian Building Materials, No. 2, pp. 59-61.

[10] Zhang J.S. (2008). Self-Thermal insulation system of external wall and construction energy saving in the areas of hot summer and cold winter, Housing Science, No. 4, pp. 6-7.

[11] Gu L.J., Yu C. (2011). Status of building energy use data and analysis of energy use statistics in China, Energy of China, Vol. 33, No. 2, pp. 38-41. DOI: 10.3969/j.issn.1003-2355.2011.02.008

[12] Yu W.Z., Kang W.B. (2011). Developing selfinsulation block of lightweight concrete, Building Block and Block Building, No. 1, pp. 18-21. DOI: 10.3969/j.issn.1003-5273.2011.01.004

[13] Song Y.L. (2011). Research on energy saving by $65 \%$ and self-thermal insulation blocks in cold regions, China Concrete and Cement Products, No. 7, pp. 4750. DOI: $10.3969 /$ j.issn.1000-4637.2011.07.013

[14] Ergun A. (2011). Effects of the usage of diatomite and waste marble powder as partial replacement of cement on the mechanical properties of concrete, Construction \& Building Materials, Vol. 25, No. 2, pp. 806-812. DOI: $10.1016 /$ j.conbuildmat.2010.07.002

[15] Song Y.L. (2012). Study on the thermal performance of concrete self-insulation masonry wall, China Concrete and Cement Products, No. 2, pp. 52-56. DOI: $\underline{10.3969 / \text { j.issn.1000-4637.2012.02.014 }}$ 